\title{
A Cirurgia de Substituição Valvular Aórtica Melhora a Qualidade de Vida dos Doentes?
}

\section{Does Aortic Valve Replacement Surgery Improve Patients' Quality of Life?}

\author{
Pedro COELHO ${ }^{1,2}$, Lara N. FERREIRA $\varangle^{3,4}$, Clara VITAL ${ }^{1}$, José FRAGATA ${ }^{1,2}$ \\ Acta Med Port 2018 Jul-Aug;31(7-8):399-408 - https://doi.org/10.20344/amp.10241
}

\section{RESUMO}

Introdução: O objetivo da cirurgia de substituição valvular aórtica é o aumento da esperança de vida e a melhoria da qualidade de vida relacionada com a saúde. Existem vários estudos que evidenciam melhoria da qualidade de vida relacionada com a saúde nestes doentes, mas não aplicados à população portuguesa. O objetivo deste estudo é avaliar a qualidade de vida relacionada com a saúde pós-implantação de prótese aórtica e comparar com a qualidade de vida relacionada com a saúde pré-operatória e da população em geral.

Material e Métodos: Foi feito um estudo retrospetivo de doentes eletivos submetidos a implantação de prótese aórtica entre agosto de 2011 e abril de 2016. A qualidade de vida relacionada com a saúde foi avaliada com o Short Form 36 Health Survey Instrument e com o Short Form 6 Health Survey Instrument no pré-operatório e aos 3, 6 e 12 meses pós-cirurgia. Foram realizadas análises descritivas e inferenciais para analisar globalmente a amostra e para comparar a qualidade de vida relacionada com a saúde pré-operatória com a pós-operatória e com as normas da população portuguesa.

Resultados: Foram incluídos 506 doentes, com idade média de 70,6 anos, sendo 53,6\% do sexo masculino. Os resultados do pósoperatório evidenciam uma melhoria estatisticamente significativa quando comparados com o pré-operatório nas oito dimensões do Short Form 36 Health Survey Instrument. Comparando com a população Portuguesa em geral, a qualidade de vida relacionada com a saúde dos doentes é inferior no pré-operatório, melhorando no pós-operatório, atingindo níveis de qualidade de vida relacionada com a saúde superiores nalguns subgrupos de doentes e em algumas dimensões. Os resultados do Short Form 6 Health Survey Instrument revelam maior intensidade de problemas no pré-operatório quando comparados com a população em geral, aproximando-se dos valores população em geral ao final de um ano pós-cirurgia.

Discussão: Este é o primeiro estudo realizado em Portugal que compara a qualidade de vida relacionada com a saúde, usando uma escala validada, antes e depois da cirurgia de implantação de prótese aórtica. Os resultados demonstram a existência de uma melhoria significativa em todas as dimensões, o que não acontece consistentemente noutros estudos publicados.

Conclusão: A implantação de prótese aórtica melhora a qualidade de vida relacionada com a saúde dos doentes, permitindo que esta se aproxime dos resultados da população em geral.

Palavras-chave: Implante de Prótese de Válvulas Cardíacas; Inquéritos e Questionários; Qualidade de Vida; Válvula Aórtica/cirurgia

\section{ABSTRACT}

Introduction: Aortic valve replacement surgery is done to increase patients' life expectancy and improve their health-related quality of life. Several published studies have found improvement in health-related quality of life after this procedure, but none have involved patients in Portugal. This study sought to evaluate patients' health-related quality of life after the implantation of aortic prostheses and compare these values with preoperative health-related quality of life and the general Portuguese population's health-related quality of life.

Material and Methods: A retrospective study was done with elective patients who underwent implantation of an aortic prosthesis between August 2011 and April 2016. Health-related quality of life was evaluated using the Short Form 36 Health Survey and Short Form 6 Health Survey questionnaires in the preoperative period and 3, 6 and 12 months post-surgery. Descriptive and inferential analyses were conducted to process the data and compare preoperative health-related quality of life with postoperative values and the Portuguese population's norms.

Results: The sample included 506 patients with an average age of 70.6 years. The majority are male (53.6\%). The postoperative results show a statistically significant improvement compared to preoperative health-related quality of life in all eight dimensions of the Short Form 36 Health Survey. When contrasted with the general Portuguese population, patients' health-related quality of life is lower in the preoperative period, improving postoperatively and reaching higher than average health-related quality of life levels in some subgroups of patients and in various dimensions. An analysis of the Short Form 6 Health Survey results revealed that the patients undergoing this surgery have a higher level of preoperative problems compared with the general population, but these patients' values approach those of the general population by the end of one year post-surgery.

Discussion: This is the first study in Portugal that compares health-related quality of life using a validated scale, before and after the aortic prosthesis implantation surgery. The results demonstrate a significant improvement in all dimensions, which is not consistently observed in other published studies.

Conclusion: The results confirm that the implantation of aortic prostheses improves patients' health-related quality of life, over time bringing it close to the general population's levels.

Keywords: Aortic Valve/surgery; Heart Valve Prosthesis Implantation; Quality of Life; Surveys and Questionnaires

1. Serviço de Cirurgia Cardiotorácica. Hospital de Santa Marta. Centro Hospitalar de Lisboa Central. Lisboa. Portugal.

2. Faculdade de Ciências Médicas. Universidade Nova de Lisboa. Lisboa. Portugal.

3. Escola Superior de Gestão, Hotelaria e Turismo. Universidade do Algarve. Faro. Portugal.

4. Centro de Estudos e Investigação em Saúde. Universidade de Coimbra. Coimbra. Portugal.

$\square$ Autor correspondente: Lara N. Ferreira. Inferrei@ualg.pt

Recebido: 14 de janeiro de 2018 - Aceite: 15 de maio de 2018 | Copyright @ Ordem dos Médicos 2018 


\section{INTRODUÇÃO}

A estenose aórtica é uma doença cuja incidência vai aumentando progressivamente com a idade, indo de $0,02 \%$ na população dos 18 aos 44 anos até aos $2,8 \%$ naqueles com mais de 75 anos. ${ }^{1}$ Os sintomas desta doença são angina, dispneia e sincope. ${ }^{2} \mathrm{O}$ aparecimento destes sintomas está diretamente relacionado com a sobrevida média, sendo respetivamente de 5,3 e 2 anos. ${ }^{3} \mathrm{O}$ tratamento num grande número de doentes com este tipo de patologia é a substituição valvular. A cirurgia tem como objetivo o aumento da sobrevida e a melhoria da qualidade de vida destes doentes ${ }^{4} \mathrm{~A}$ grande maioria dos doentes submetidos a este tipo de cirurgia são idosos, tendo muitos deles já atingido a sua esperança de vida, pelo que os ganhos em qualidade de vida relacionada com a saúde (QVRS) assumem maior importância.

Nos últimos anos tem-se verificado um aumento da importância do estudo da QVRS de doentes cardíacos, ${ }^{5-9}$ usando instrumentos genéricos como o Short Form 36 Health Survey Instrument (SF-36) ou o EuroQol (EQ-5D). Na realidade, o estudo da QVRS de doentes submetidos a esta cirurgia tem sido o foco de vários artigos, ${ }^{10-14}$ mas poucos estudos têm sido realizados em Portugal ${ }^{15,16} \mathrm{e}$ em nenhum é realizada uma comparação com a população portuguesa, o que permitiria identificar e valorizar o impacto da doença nestes doentes.

Neste trabalho pretendeu-se medir a QVRS dos doentes submetidos a implantação de prótese valvular aórtica num centro cirúrgico português no período 2011 a 2016.

\section{MATERIAL E MÉTODOS Desenho do estudo}

Foi conduzido um estudo retrospetivo com dados recoIhidos prospectivamente no Serviço de Cirurgia Cardiotorácica do Hospital de Santa Marta. Foram incluídos os doentes submetidos a cirurgia de implantação de prótese aórtica entre agosto de 2011 e abril de 2016. Foram excluídos os doentes urgentes e emergentes, bem como os doentes internados noutros hospitais previamente à cirurgia ou que, por dificuldades de linguagem, não conseguiram responder ao questionário. Foram ainda excluídos os doentes submetidos a outro procedimento para além da implantação de prótese aórtica no mesmo tempo cirúrgico. O SF-36 v2 foi aplicado aos doentes na véspera da intervenção cirúrgica presencialmente e depois nas consultas de seguimento aos 3, 6 e 12 meses após a cirurgia. Nos casos em que as consultas se realizaram com uma diferença de mais de 15 dias das datas anteriormente referidas ou os doentes não compareceram às mesmas, os questionários foram aplicados por telefone. A aplicação dos questionários foi realizada pelas enfermeiras do serviço, responsáveis pelo acolhimento pré-cirúrgico do doente. Nos casos em que foi necessário aplicá-los por telefone, a aplicação foi realizada pela enfermeira encarregue do follow-up nesse dia.

Foram explicados aos doentes os objetivos do estudo, nomeadamente que os questionários são aplicados por rotina a todos os doentes submetidos a cirurgia no serviço e que os resultados permitem monitorizar a evolução do seu estado de saúde. Todos os doentes preencheram um documento de consentimento informado.

\section{Medidas}

A auto-perceção do estado de saúde e a QVRS dos doentes foram medidas, respetivamente, pelos questionários Short Form 36 Health Survey (SF-36v2) e pelo Short Form 6 Health Survey (SF-6D).

\section{Short Form 36 Health Survey - SF-36v2}

O SF-36, com 36 questões, mede oito dimensões em saúde, numa escala de 0 a 100, com os extremos a corresponderem, respetivamente, à pior e à melhor QVRS possíveis. As dimensões medidas incluem a função física (FF), as limitações de desempenho devido a problemas físicos (DF) ou emocionais (DE), a intensidade e o desconforto causado pela dor (D), a saúde em geral (SG), a vitalidade (VT), a função social (FS) e a saúde mental (SM). ${ }^{17,18}$ Uma explicação detalhada das componentes de cada dimensão pode ser encontrada em Ferreira e colegas. ${ }^{19}$

As oito dimensões do SF-36 podem ser agregadas em duas medidas sumário física e mental ${ }^{20}$. A medida sumário física (MSF) compreende as dimensões FF, DF, D e SG, e a medida sumário mental (MSM) é constituída pelas dimensões SM, DE, FS e VT. Este instrumento é utilizado em todo o mundo, tanto na população em geral, ${ }^{21,22} \mathrm{como} \mathrm{em}$ subgrupos da população ${ }^{23}$ ou em grupos de doentes. ${ }^{24,25}$ Tem vindo a ser alvo de inúmeras traduções e validações. A primeira versão deste instrumento foi validada para a população portuguesa no início deste século. 26,27 Posteriormente, a versão original do SF-36 foi modificada pelos autores para ajustar a utilização do SF-36 a novas línguas e culturas, tendo sido criada a segunda versão do SF-36 (SF-36v2). ${ }^{28}$ Assim, em 2003 foi criada a segunda versão em português e as normas portuguesas para esta versão. ${ }^{29}$ Esta versão SF-36 (SF-36v2), bem como as suas normas, foram obtidas com base numa amostra da população portuguesa $(n=2549)$ que respondeu ao SF-36v2 e a questões de natureza sociodemográfica. Os dados foram recolhidos em 1999 por entrevista pessoal nas residências dos respondentes.

Diversos estudos realçaram a importância da existência de valores normativos ou normas da população que possam ser usados como dados de referência para interpretar os valores obtidos num dado estudo. ${ }^{21,30}$ Esses dados permitem comparar perfis de doentes com condições específicas, com dados de um indivíduo comum da população em geral, pertencente a um grupo etário e/ou género semelhante. ${ }^{31,32}$ Estes tipos de comparações permitem, por exemplo, a identificação do fardo da doença numa população em particular, com uma determinada doença. Neste sentido, os resultados reportados neste estudo foram comparados com as normas da população portuguesa relativas ao SF-36v $2^{29}$ e com as normas relativas às medidas sumário física e mental do SF-36v2. ${ }^{19}$ As normas relativas 
às medidas sumário física e mental do SF-36 estão publicadas em Ferreira e colegas. ${ }^{19}$ Os dados foram recolhidos através de entrevistas telefónicas realizadas entre 2011 e 2012 a uma amostra aleatória da população portuguesa estratificada por idade, género e região NUTS II ( $n=1500)$. Para além da versão portuguesa do SF-36v2, foram recoIhidos dados de natureza sociodemográfica, e informação relativa a doenças que tivessem sido comunicadas aos respondentes por um médico ou outro profissional de saúde.

\section{Short Form 6 Health Survey - SF-6D}

O SF-6D é um índice econométrico baseado em preferências que resulta de 11 itens do SF-36 convertidos num sistema de classificação com seis dimensões, cada uma delas com quatro a seis níveis, permitindo gerar um total de 18000 estados de saúde diferentes. ${ }^{33}$ Foi desenvolvido com o objetivo de permitir a utilização em valorações de um dos perfis de saúde mais utilizados em todo o mundo, o SF36 , isto é, de o tornar também num instrumento de medição de preferências. Os autores utilizaram uma amostra representativa da população do Reino Unido que atribuiu valores a 249 estados de saúde definidos pelo SF-6D, através do jogo padrão. ${ }^{33}$ Esses valores permitiram estimar modelos econométricos, a partir dos quais se atribuíram pesos aos diferentes níveis das dimensões do SF-6D, gerando-se valores para todos os estados de saúde definidos pelo SF-6D. Estes valores dos estados de saúde constituem um índice, o índice SF-6D, que pode ser visto como um valor contínuo numa escala de 0,35 a 1,00. A popularidade do SF-6D advém do facto de ser obtido a partir do SF-36, pelo que pode ser utilizado em todas as línguas para as quais o SF-36 (v1 ou v2) esteja oficialmente traduzido e validado. Nos últimos anos, foram determinados sistemas de valores para vários países, ${ }^{34-38}$ existindo desde 2006 a versão portuguesa do SF-6D ${ }^{39}$ e desde 2010 o sistema português de valores para o SF-6D. ${ }^{40}$

A existência de normas para o SF-6D permite a análise dos efeitos dos cuidados de saúde e outras intervenções, a determinação do fardo da doença e a realização de comparações, seja de subgrupos da população com a popula- ção em geral, seja comparações regionais e entre países. No caso do SF-6D existem dados normativos publicados para diferentes países, como o Reino Unido, ${ }^{41}$ a Austrália ${ }^{30}$ e Portugal. ${ }^{42}$ Uma amostra aleatória e representativa da polução portuguesa $(n=1500)$ respondeu ao SF-36v2 por entrevista telefónica. O sistema de valores do SF-6D foi usado para obter o índice SF-6D e assim obter as normas para o SF-6D da população portuguesa. Mais detalhes do desenho do estudo e da amostra podem ser encontrados em Ferreira e colegas. ${ }^{42}$

\section{Análise de dados}

Foram aplicadas análises descritivas e inferenciais às variáveis sociodemográficas, às dimensões do SF-36v2, às componentes física (MSF) e mental (MSM) do SF-36v2, às dimensões do SF-6D e ao índice SF-6D. A análise descritiva consistiu no cálculo de distribuições de frequências e medidas de estatística descritiva. Foram também utilizados gráficos para melhor visualizar a comparação das estimativas da média das dimensões do SF-36v2 e da MSF e da MSM dos doentes com as da população portuguesa. A análise inferencial incidiu na utilização de testes paramétricos (testes $t$ e ANOVA) e não paramétricos (Wilcoxon e Kruskal Wallis), de acordo com as características da distribuição em análise. Estas técnicas foram usadas para analisar globalmente a amostra de doentes e comparar a QVRS dos doentes antes da cirurgia e após a cirurgia nos momentos de seguimento subsequentes (três e seis meses e um ano). O estado de saúde auto-reportado e os resultados da QVRS das amostras de doentes também foram comparados com as normas para a população portuguesa em geral. ${ }^{19,29,42}$ Para calcular as componentes MSF e MCS do SF-36v2 foram utilizadas as expressões determinadas para a população portuguesa em Ferreira e colegas. ${ }^{19} \mathrm{O}$ cálculo do índice SF-6D foi realizado utilizando o sistema de valores português. ${ }^{40}$

Os dados foram analisados com o programa estatístico IBM SPSS Statistics, versão 24. O nível de significância foi fixado em $5 \%$ para todas as análises estatísticas.

Tabela 1 - Características da amostra inicial, nos momentos de seguimento e da população portuguesa

\begin{tabular}{|c|c|c|c|c|c|}
\hline & $\begin{array}{c}\text { Amostra inicial } \\
(n=506)\end{array}$ & $\begin{array}{l}\text { Seguimento } \\
\text { a } 3 \text { meses } \\
(n=458)\end{array}$ & $\begin{array}{l}\text { Seguimento } \\
\text { a } 6 \text { meses } \\
(n=457)\end{array}$ & $\begin{array}{c}\text { Seguimento } \\
\text { a } 1 \text { ano } \\
(n=442)\end{array}$ & $\begin{array}{c}\text { População Portuguesa } \\
\geq 18 \text { anos }^{43} \\
(n=8657240)\end{array}$ \\
\hline Homens (\%) & 53,6 & 53,3 & 53,8 & 53,6 & 47,0 \\
\hline \multicolumn{6}{|l|}{ Idade (na cirurgia) } \\
\hline $18-29$ anos (\%) & 0,4 & 0,5 & 0,5 & 0,5 & 17,0 \\
\hline $30-49$ anos (\%) & 5,6 & 5,9 & 6,2 & 5,9 & 36,3 \\
\hline $50-69$ anos $(\%)$ & 30,0 & 31,1 & 31,7 & 32,4 & 29.9 \\
\hline+70 anos $(\%)$ & 64,0 & 62,5 & 61,6 & 61,2 & 16,8 \\
\hline Média (desvio padrão) & $70,6(11,6)$ & $70,3(11,9)$ & $70,1(11,9)$ & $70,0(11,7)$ & $49,4(18,5)$ \\
\hline Altura média* (desvio padrão) & $162,5(8,9)$ & $162,3(8,9)$ & $162,4(9,0)$ & $162,3(8,9)$ & n.d. \\
\hline Peso médio** (desvio padrão) & $73,3(13,6)$ & $72,8(13,4)$ & $73,2(13,5)$ & $72,9(13,4)$ & n.d. \\
\hline
\end{tabular}




\section{RESULTADOS}

\section{Amostra}

A Tabela 1 sumariza as principais características da amostra, no momento inicial e nos três momentos de seguimento. É também apresentada a mesma informação para a população portuguesa com idade igual ou superior a 18 anos para efeitos de comparação. ${ }^{43} \mathrm{~A}$ amostra foi inicialmente constituída por 506 doentes, sendo a maioria do sexo masculino. A maioria dos doentes tinha mais de 50 anos de idade, tendo uma média de idades de 70,6 anos. No que diz respeito ao peso, em média os doentes pesavam cerca de $73 \mathrm{~kg}$ e mediam $162,5 \mathrm{~cm}$. Três meses depois da cirurgia, foram avaliados 458 doentes, seis meses depois 457 e 1 ano após a cirurgia 442, o que significa que dos doentes iniciais cerca de $87 \%$ foram seguidos até um ano depois. Não foi possível seguir todos os doentes inicialmente incluídos no estudo porque alguns morreram e outros não compareceram às consultas ou, tendo comparecido, não Ihe foi entregue o questionário para preenchimento. Em alguns destes casos os questionários foram aplicados por telefone; no entanto, em algumas situações não foi possível estabelecer ou realizar a chamada dentro do tempo de seguimento estipulado no protocolo do estudo. Como seria de esperar, as características das amostras nos três momentos de seguimento são bastantes semelhantes à da amostra inicial (Tabela 1).

Na Tabela 2 são apresentadas as principais características da amostra de doentes operados, tendo em conta as suas comorbilidades, tipo de prótese colocada, tempo de permanência na unidade de cuidados intensivos, tempo de internamento, complicações pós-operatórias e mortalidade ao final de 1 mês e de 1 ano. Em termos de comorbilidades, verifica-se que uma percentagem elevada de doentes sofria de hipertensão arterial, hipercolesterolemia e

Tabela 2 - Comorbilidades dos doentes operados $(n=506)$, tipo de prótese, tempo de internamento, complicações pós-operatórias e mortalidade

\begin{tabular}{|c|c|c|c|}
\hline & & Número / média (desvio padrão) & $\%$ \\
\hline Índice massa corporal & & $28,3(8,0)$ & - \\
\hline \multirow[t]{5}{*}{ Angina } & $\operatorname{ccs} 0$ & 104 & 20,6 \\
\hline & $\operatorname{ccs} I$ & 259 & 51,2 \\
\hline & CCS II & 111 & 21,9 \\
\hline & CCS III & 30 & 5,9 \\
\hline & CCS IV & 2 & 0,4 \\
\hline \multirow[t]{4}{*}{ Classe New York Heart Association } & NYHA I & 15 & 29,6 \\
\hline & NYHA II & 207 & 40,9 \\
\hline & NYHA III & 275 & 54,3 \\
\hline & NYHA IV & 9 & 1,8 \\
\hline Hipertensão arterial & & 435 & 86 \\
\hline Hipercolesterolemia & & 368 & 72,8 \\
\hline Diabetes & & 347 & 68,6 \\
\hline Doença pulmonar obstrutiva crónica & & 49 & 9,7 \\
\hline Creatinina pré-operatória & & $1,0(0,5)$ & - \\
\hline \multirow[t]{3}{*}{ Fracção de ejecção } & $<30 \%$ & 10 & 2 \\
\hline & $30 \%-49 \%$ & 91 & 18 \\
\hline & $>49 \%$ & 405 & 80 \\
\hline EUROSCORE I & & $6,8(5,1)$ & - \\
\hline \multirow[t]{2}{*}{ Tipo de prótese } & Biológica & 404 & 79,8 \\
\hline & Mecânica & 102 & 20,2 \\
\hline Tempo de internamento na unidade de cuidados intensivos & & $2,7(2,2)$ & - \\
\hline Acidente vascular cerebral & & 8 & 1,6 \\
\hline Ventilação mais 24 horas & & 17 & 3,4 \\
\hline Suporte inotrópico mais de 24 horas & & 140 & 27,7 \\
\hline Fibrilhação auricular & & 92 & 18,2 \\
\hline Transfundidos & & 345 & 68,2 \\
\hline Tempo de Internamento & & $9,6(7,1)$ & - \\
\hline Mortalidade ao final de $\mathbf{3 0}$ dias & & 6 & 1,2 \\
\hline Mortalidade ao final de um ano & & 35 & 6,9 \\
\hline
\end{tabular}

CCS: Canadian Cardiovascular Surgery 
diabetes. Quanto à gravidade da angina, considerando a classe Canadian Cardiovascular Surgery (CCS), a maioria dos doentes está na classe I e II. Quanto à insuficiência cardíaca, considerando a classe de insuficiência cardíaca da New York Heart Association (NYHA), a maioria está na classe II e III. Esta distribuição justifica-se pela maior frequência de insuficiência cardíaca neste tipo de patologia. A maioria das próteses implantadas são biológicas, o que é justificado pela maior parte dos doentes serem idosos com mais de 65 anos. Quanto às complicações pós-operatórias descritas, a sua incidência encontra-se dentro dos valores expectáveis. Foi realizada uma estratificação de risco com o EUROSCORE I, escala que prevê a mortalidade aos 30 dias, tendo a mortalidade verificada aos 30 dias sido inferior à prevista pela escala. No total, verificaram-se 41 mortes (ao final de 30 dias e de 1 ano). Estes doentes falecidos representam $64 \%$ dos doentes que não foram seguidos até ao final do estudo.

\section{Evolução da qualidade de vida relacionada com a saú-} de e comparação com as normas da população

A Fig. 1 compara as estimativas da média das dimensões do SF-36 dos doentes antes e um ano após a cirurgia com a população portuguesa. É possível observar que para todas as dimensões se verificou um aumento após a cirurgia do estado de saúde percebido pelos doentes. Quando comparadas com a população portuguesa, verifica-se que nas dimensões físicas (função física e desempenho físico) os valores reportados pelos doentes após a cirurgia se assemelham aos da população portuguesa. Pelo contrário, na dor e saúde mental, embora se verifique uma melhoria, os valores dos doentes após a cirurgia ainda são bastante inferiores à população portuguesa.

Na Fig. 2 são comparadas as estimativas da média da MSF do SF-36v2 com a população portuguesa. Como seria de esperar após a cirurgia dos doentes os valores aproximam-se dos da população portuguesa para o total da amostra. Efetuando as comparações por género verifica-se que os homens apresentam uma estimativa da MSF após a cirurgia um pouco superior à população portuguesa. Quando se realizam as comparações por classe etária, verifica-se que a partir dos 30 anos de idade, as estimativas da média da MSF do SF-36v2 após a cirurgia são sempre superiores às da população portuguesa. Apenas na classe etária dos indivíduos mais jovens, a média da MSF se apresenta um pouco inferior à da população portuguesa.

A Fig. 3 permite-nos verificar que, à semelhança do que se verificou com a MSF, os doentes um ano após a cirurgia apresentam valores médios superiores aos que se verificavam antes da cirurgia, tanto quando se considera a totalidade da amostra, como quando se analisa por género e classe etária, sendo essas diferenças estatisticamente significativas. É de realçar que os valores após a cirurgia são superiores aos da população portuguesa para a totalidade da amostra, para ambos os géneros e para todas as classes etárias, com exceção dos indivíduos com menos de 30 anos de idade.

No que diz respeito à QVRS (Tabela 3), o sistema descritivo do SF-6D mostra, para cada nível e dimensão, que os doentes com necessidade de substituição valvular aórtica têm maior intensidade de problemas quando comparados com a população portuguesa, ${ }^{42}$ sendo essas diferenças

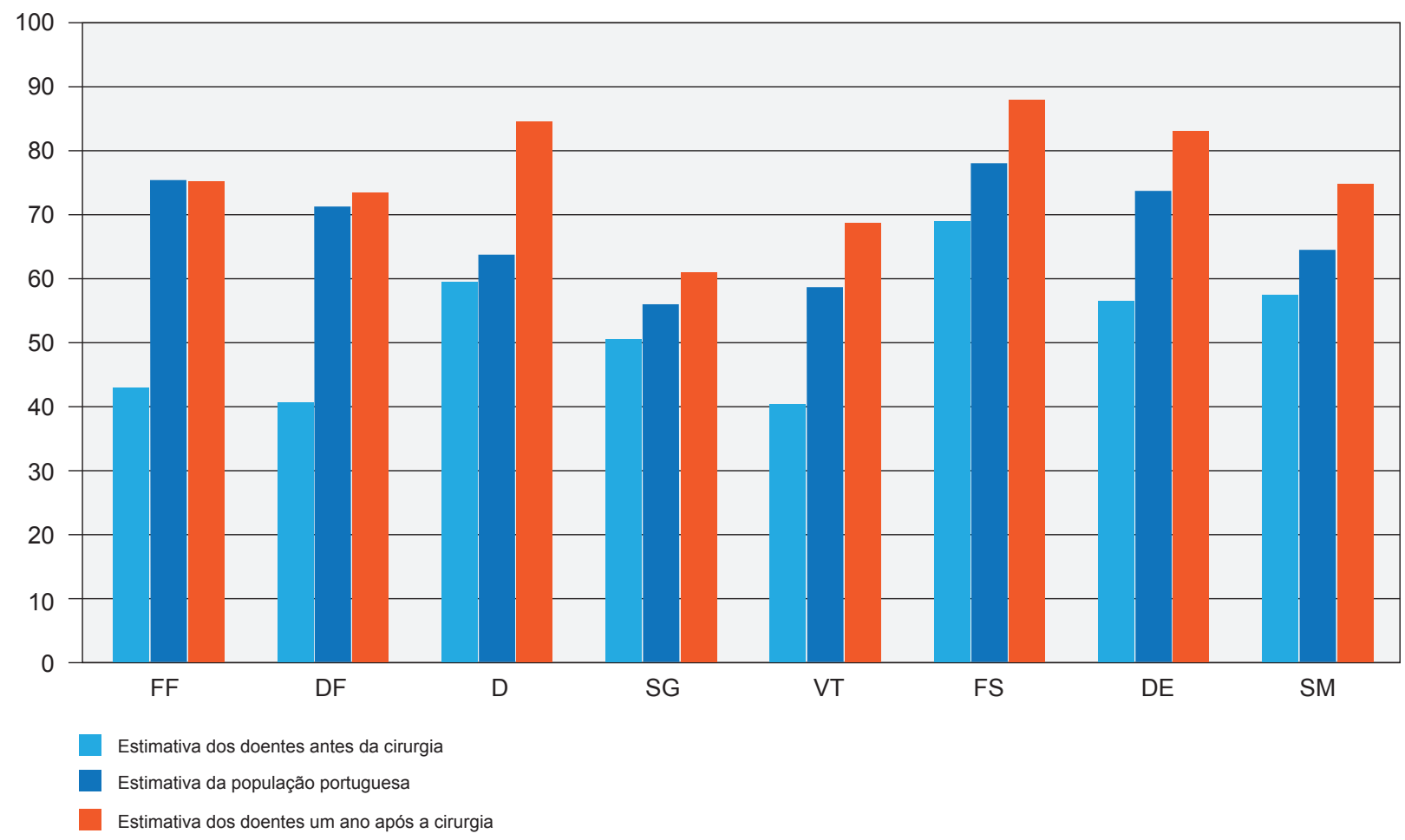

Figura 1 - Comparação das estimativas da média das dimensões do SF-36 dos doentes antes e um ano após a cirurgia com a população portuguesa $^{29}$

FF: função física; DF: desempenho físico; D: dor; SG: saúde geral; VT: vitalidade; FS: função social; DE: desempenho emocional; SM: saúde mental. 


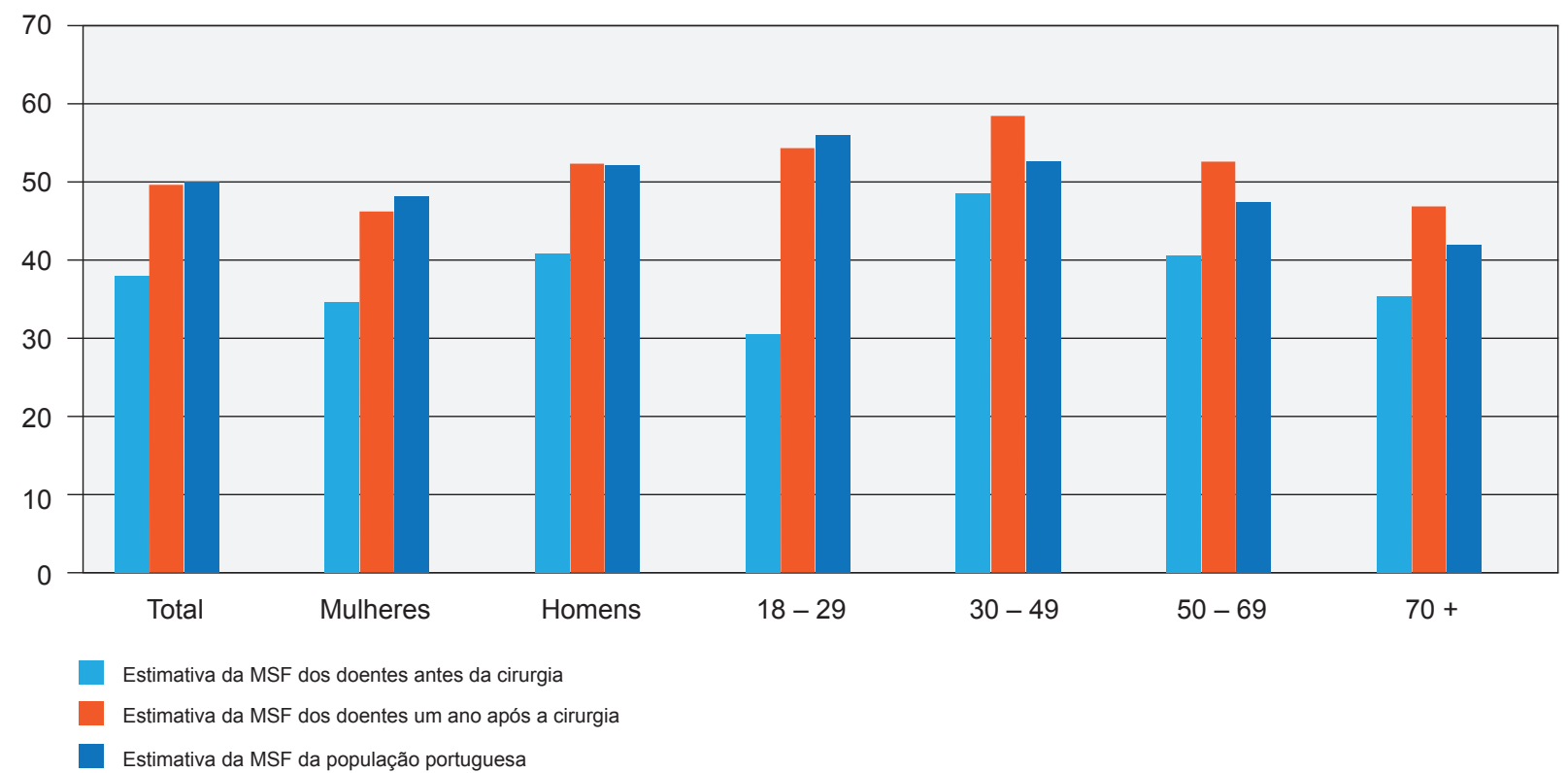

Figura 2 - Comparação das estimativas da média da componente física (MSF) do SF-36v2 dos doentes antes e um ano após a cirurgia com a população portuguesa ${ }^{29}$ por género e grupo etário

estatisticamente significativas. Os problemas reportados são intensos em todas as dimensões, sendo menos intensos na dimensão função social. As melhorias são evidentes em todas as dimensões. O índice SF-6D variou entre 0,38 e 1,00 , com uma média de 0,75 , inferior à da população portuguesa. Com a cirurgia, a QVRS dos doentes aumentou, sendo que após um ano a média do índice SF-6D coincide com a da população portuguesa. Note-se que as diferenças são estatisticamente significativas quando se compara o índice SF-6D a 3 meses, 6 meses e 1 ano com o momento inicial e quando se compara a evolução ao longo do tempo (6 meses com 3 meses e 1 ano com 3 meses).

A Fig. 4 mostra a evolução do índice SF-6D por género e classe etária e compara esses valores com a população portuguesa. Como seria de esperar, os homens apresentam uma QVRS superior à das mulheres. Em ambos os géneros a QVRS após a cirurgia é mais elevada, aproximando-se dos valores da população portuguesa. No que diz respeito à idade, a QVRS diminui com o aumento da idade a partir dos 30 anos, mas, como seria de esperar, após a cirurgia a QVRS é superior em todas as classes etárias, chegando mesmo a ser superior do que a da população portuguesa. A exceção é a classe etária mais jovem, pois os indivíduos entre 18 e 29 anos de idade apresentam uma QVRS muito baixa antes da cirurgia, talvez porque considerem que o problema que têm os limita grandemente nas suas atividades. Um ano após a cirurgia verifica-se que a QVRS desses indivíduos aumentou bastante, aproximando-se

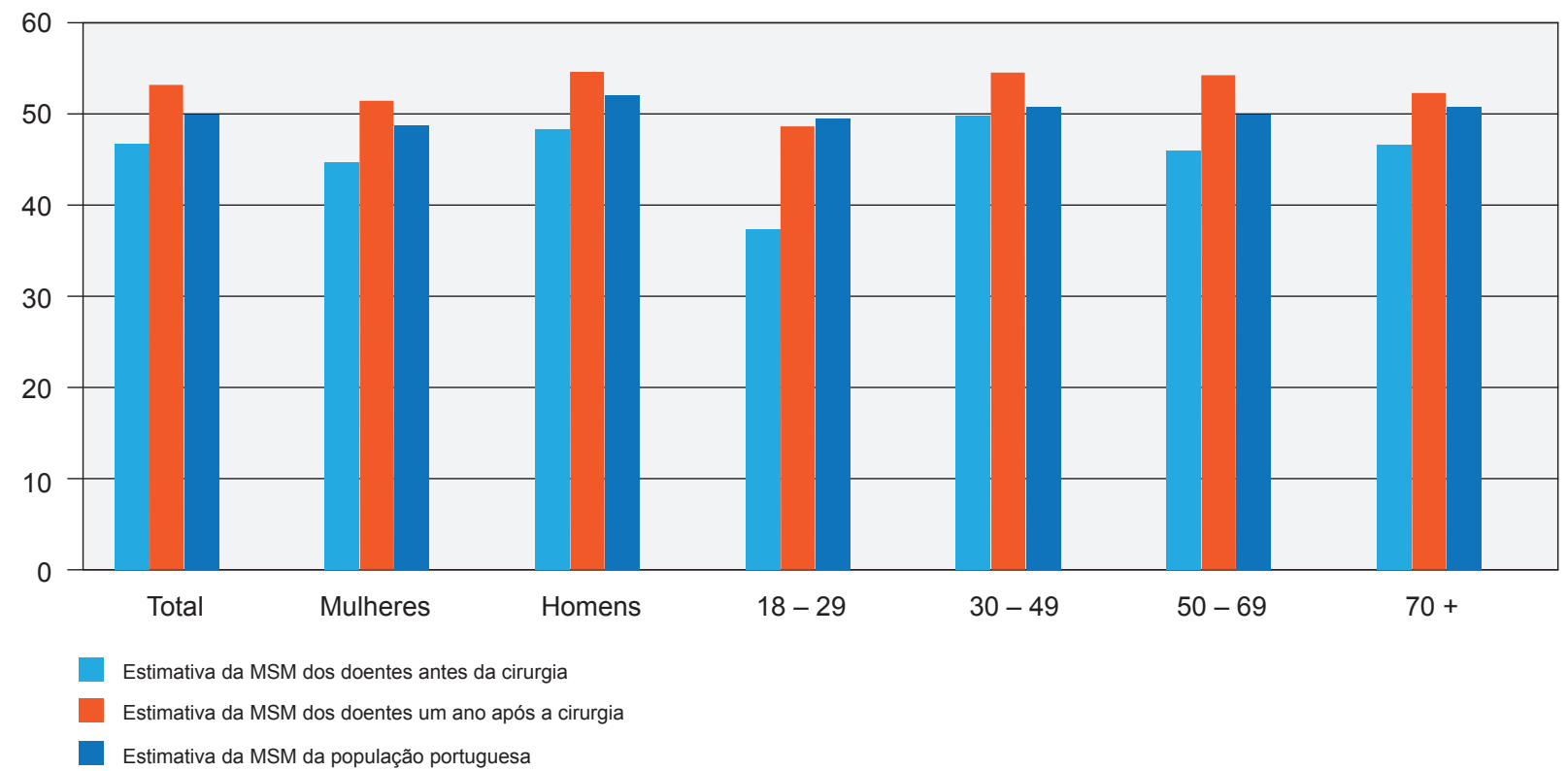

Figura 3 - Comparação das estimativas da média da componente mental (MSM) do SF-36v2 dos doentes antes e um ano após a cirurgia com a população portuguesa ${ }^{29}$ por género e grupo etário 
Tabela 3 - Estimativas da população relativamente às dimensões do SF-6D (\%)

\begin{tabular}{|c|c|c|c|c|c|c|}
\hline $\begin{array}{l}\text { Dimensões do } \\
\text { SF-6D / níveis }\end{array}$ & & $\begin{array}{c}\text { Amostra } \\
\text { inicial } \\
(n=506)\end{array}$ & $\begin{array}{c}\text { Seguimento a } \\
3 \text { meses } \\
(n=458)\end{array}$ & $\begin{array}{l}\text { Seguimento a } \\
6 \text { meses } \\
(n=457)\end{array}$ & $\begin{array}{c}\text { Seguimento a } \\
1 \text { ano } \\
(n=442)\end{array}$ & $\begin{array}{l}\text { População } \\
\text { Portuguesa }{ }^{42}\end{array}$ \\
\hline \multirow[t]{6}{*}{ Função física } & 1 & 4,3 & 4,4 & 9,0 & 12,7 & 37,8 \\
\hline & 2 & 5,3 & 17,7 & 19,5 & 23,5 & 31,2 \\
\hline & 3 & 26,9 & 42,0 & 38,9 & 33,0 & 18,1 \\
\hline & 4 & 25,5 & 27,6 & 24,3 & 20,6 & 2,9 \\
\hline & 5 & 28,5 & 5,7 & 6,3 & 8,1 & 7,9 \\
\hline & 6 & 9,5 & 2,6 & 2,0 & 2,0 & 2,2 \\
\hline \multirow{4}{*}{$\begin{array}{l}\text { Limitação do } \\
\text { desempenho }\end{array}$} & 1 & 10,5 & 24,3 & 34,4 & 39,9 & 44,8 \\
\hline & 2 & 12,6 & 26,8 & 25,6 & 21,2 & 12,0 \\
\hline & 3 & 4,5 & 2,0 & 2,2 & 1,8 & 13,8 \\
\hline & 4 & 72,3 & 46,9 & 37,9 & 37,1 & 29,4 \\
\hline \multirow[t]{5}{*}{ Função social } & 1 & 30,4 & 59,9 & 64,3 & 67,2 & 55,8 \\
\hline & 2 & 20,6 & 26,4 & 21,0 & 19,1 & 15,5 \\
\hline & 3 & 26,5 & 9,0 & 10,1 & 7,3 & 18,7 \\
\hline & 4 & 15,4 & 3,3 & 2,8 & 4,3 & 6,8 \\
\hline & 5 & 7,1 & 1,3 & 1,8 & 2,1 & 3,2 \\
\hline \multirow[t]{6}{*}{ Dor } & 1 & 21,9 & 48,2 & 56,6 & 58,7 & 31,6 \\
\hline & 2 & 10,1 & 16,5 & 14,0 & 10,7 & 19,5 \\
\hline & 3 & 24,1 & 24,0 & 19,5 & 18,9 & 21,0 \\
\hline & 4 & 21,5 & 7,9 & 7,2 & 8,9 & 17,4 \\
\hline & 5 & 17,2 & 2,4 & 2,0 & 1,6 & 7,4 \\
\hline & 6 & 5,1 & 0,9 & 0,7 & 1,1 & 3,1 \\
\hline \multirow[t]{5}{*}{ Saúde mental } & 1 & 9,9 & 21,1 & 30,6 & 26,5 & 25,0 \\
\hline & 2 & 18,6 & 43,5 & 39,8 & 39,2 & 24,6 \\
\hline & 3 & 37,4 & 25,1 & 21,2 & 23,8 & 32,2 \\
\hline & 4 & 23,3 & 8,8 & 7,2 & 9,3 & 10,1 \\
\hline & 5 & 10,9 & 1,5 & 1,1 & 1,1 & 8,1 \\
\hline \multirow[t]{5}{*}{ Vitalidade } & 1 & 4,7 & 4,6 & 9,0 & 8,6 & 20,9 \\
\hline & 2 & 15,8 & 32,3 & 35,7 & 42,6 & 32,7 \\
\hline & 3 & 24,3 & 34,9 & 35,0 & 27,2 & 27,0 \\
\hline & 4 & 33,4 & 20,4 & 14,7 & 16,8 & 12,6 \\
\hline & 5 & 21,7 & 7,7 & 5,7 & 4,8 & 6,8 \\
\hline \multicolumn{2}{|c|}{ Média do índice SF-6D (DP) } & $0,75(0,11)$ & $0,84(0,08)^{*}$ & $0,85(0,08)^{*}$ & $0,85(0,09)^{*}$ & 0,85 \\
\hline
\end{tabular}

DP: desvio padrão. Nível modal assinalado a negrito. * $p<0,001$.

dos valores da população portuguesa.

\section{DISCUSSÃO}

Este estudo avalia a QVRS numa população de doentes submetidos a implantação de prótese aórtica. O questionário usado para avaliação de QVRS foi o SF-36 v2 tendo sido aplicado no pré-operatório e aos 3, 6 e 12 meses após a cirurgia. Os resultados do pós-operatório evidenciam uma melhoria estatisticamente significativa quando comparados com o pré-operatório em todas as 8 dimensões do SF-36 v2. Comparando os resultados dos doentes no pré-operatório com a população Portuguesa em geral, verifica-se que são significativamente piores. Aos 12 meses os resultados na função física e desempenho físico assemelham-se aos da população em geral, mas nas dimensões de dor e saúde mental os resultados são inferiores.

Quanto às medidas sumário componente física e mental, verificamos que a média na componente física se aproxima dos valores da população em geral, sendo até superior a esta no sexo masculino. Este resultado pode ser explicado pelo facto de a amostra ser constituída maioritariamente por idosos operados apresentando poucas patologias associadas, ao contrário da população em geral da mesma idade. Quanto à analise por idade e, mais uma vez comparando com a restante população, verificamos que, nos doentes com menos de 30 anos, a média é inferior sendo superior nos doentes acima desta idade. Estes resultados são, no entanto, difíceis de valorizar dado o reduzido 


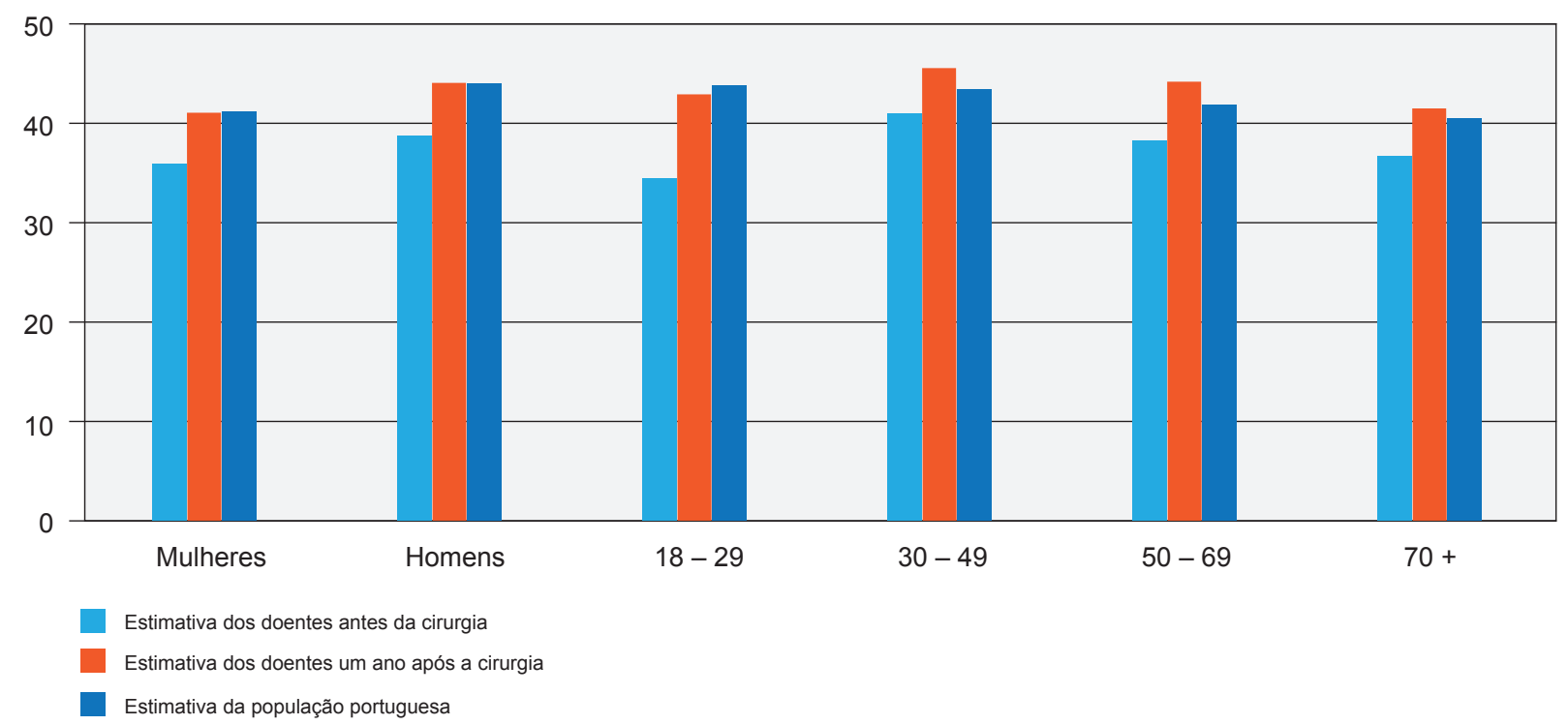

Figura 4 - Comparação das estimativas da média do índice SF-6D dos doentes antes e um ano após a cirurgia com a população portuguesa ${ }^{42}$ por género e grupo etário

número de doentes da nossa amostra com menos de 30 anos. Relativamente à componente mental os valores são superiores ao pré-operatório, e mesmo superiores aos da população em geral para ambos os géneros e para todas as classes etárias menos para indivíduos com idade inferior a 30 anos. A explicação deste facto pode estar ligada às limitações físicas provocadas pela doença serem grandes o que pode levar a que as melhoras tenham uma grande influência na saúde mental numa fase inicial após a cirurgia.

Analisando os resultados do SF-6D, verificamos que para cada nível de dimensão os doentes têm maior intensidade de problemas no pré-operatório quando comparados com a população em geral, mas ao final de um ano os valores são semelhantes aos da população em geral. Os homens apresentam melhores resultados quando comparados com as mulheres.

Evidencia-se neste estudo uma melhoria em todas as dimensões do SF-36 no pós-operatório, o que não acontece consistentemente noutros estudos feitos neste tipo de procedimento. 10,13 Pode-se, no entanto, afirmar com segurança que a substituição valvular aórtica melhora a qualidade de vida dos doentes, como comprovam os nossos resultados e de muitos outros autores..$^{12,14} \mathrm{~A}$ qualidade de vida resultante de outros procedimentos no âmbito da cirurgia tem sido também objeto de diversos estudos. No caso de cirurgia de revascularização coronária evidenciou-se que a melhoria é semelhante à da cirurgia valvular, ${ }^{44}$ embora a recuperação destes seja melhor que a dos doentes coronários. ${ }^{45} \mathrm{O}$ facto de os indivíduos do sexo masculino terem melhores resultados do que os do sexo feminino, pode ser explicado pelo facto dos resultados pré-operatorios serem inferiores nas mulheres, na maioria das dimensões, tal como acontece noutros estudos. ${ }^{46,47} \mathrm{~A}$ qualidade de vida após a cirurgia iguala ou supera a da população em geral, o que acontece neste estudo e é corroborado por outros. ${ }^{14}$ A dor após a cirurgia mantém-se como um problema, sendo superior à da população geral. A incidência de dor é elevada (cerca de $10 \%$ dos doentes), como comprovado por Gjeilo e colegas. ${ }^{11}$

Existem dois estudos que avaliam a qualidade de vida na população Portuguesa submetida a implantação de prótese aórtica, mas os seus resultados não podem ser diretamente comparados com este. Um dos estudos não utiliza um instrumento validado para avaliação da qualidade de vida, mas sim um instrumento de auto-perceção ${ }^{16}$; o outro utiliza o SF-36, mas compara dois grupos independentes, um submetido a cirurgia e o outro que aguarda cirurgia. ${ }^{15}$ O nosso estudo é assim o primeiro estudo realizado em Portugal que, utilizando uma escala validada, compara a QVRS antes e depois de cirurgia de substituição valvular aórtica.

Os resultados deste estudo evidenciam uma melhoria clara e estatisticamente significativa da qualidade de vida deste grupo de doentes para valores semelhantes aos da população em geral com a mesma idade. Atualmente existe ainda alguma relutância em considerar a cirurgia em doentes idosos uma vez que esta não vai aumentar a sua esperança de vida. No entanto considerando que a melhoria da qualidade de vida assume uma importância capital para uma população idosa cada vez mais numerosa, consideramos que este estudo poderá ser um contributo para a discussão sobre o facto de se poder considerar a cirurgia como uma opção num maior número de doentes. A decisão terapêutica neste grupo de doentes deverá no futuro tomar em consideração as nossas conclusões, podendo constituir uma ferramenta na discussão tida com os doentes sobre as opções terapêuticas e o que esperar no pós-cirurgia.

\section{Limitações}

É importante realçar que, como qualquer estudo desta natureza, este trabalho tem limitações que é necessário tomar em consideração. Uma delas está relacionada com 
o fato deste estudo avaliar um procedimento de um único centro cirúrgico que tem critérios próprios de aceitação de doentes para cirurgia e que poderá apresentar diferenças de ouros centros cirúrgicos. Outra está relacionada com a amostra. Por um lado, embora a amostra tenha uma dimensão considerável quando se analisa o número de aplicações dos questionários realizadas a cada doente, inclui um número reduzido de doentes abaixo dos 50 anos. No entanto, é de referir que na verdade a amostra incluída no estudo é na realidade um recenseamento, pois foram incluídos todos os doentes que cumpriam os critérios de inclusão definidos anteriormente naquele período de tempo e que aceitaram participar no estudo. Por último, é importante referir que nesta avaliação foram perdidos nos momentos de seguimento 48 doentes aos 3 meses (9,5\%), 49 doentes aos 6 meses $(9,6 \%)$ e 64 doentes ao final de 1 ano (12,6\%). Destes doentes, cerca de $64 \%$ doentes morreram. Foram realizados esforços para que fossem seguidos todos os doentes vivos da amostra inicial; no entanto, perderam-se 23 doentes, pelo que a amostra final foi de 442 doentes. Esta é uma limitação que é importante referir, embora se tenha verificado que as características dos doentes que não foram seguidos eram semelhantes às dos restantes doentes da amostra. Perder alguns doentes em estudos de seguimento desta natureza é natural e, comparando com estudos semelhantes em outras áreas, o número de doentes não seguidos é relativamente baixo.

\section{CONCLUSÃO}

Este estudo demonstra a importância de avaliar a QVRS em doentes submetidos a cirurgia de substituição valvular aórtica. Os resultados confirmaram que a implantação de prótese aórtica melhora a QVRS dos doentes, permitindo que esta se aproxime bastante dos resultados da população em geral.

Por outro lado, é de referir que este estudo constitui uma forte evidência sobre a importância do uso de instrumen-

\section{REFERÊNCIAS}

1. Thaden JJ, Nkomo VT, Enriquez-Sarano M. The global burden of aortic stenosis. Prog Cardiovasc Dis. 2014;56:565-71.

2. Bonow RO, Carabello B, de Leon AC Jr. Guidelines for the management of patients with valvular heart disease: executive summary. A report of the American College of Cardiology/ American Heart Association Task Force on Practice Guidelines (Committee on Management of Patients with Valvular Heart Disease). Circulation. 1998;98:1949-84.

3. Ross J, Braumwald E. Aortic stenosis. Circulation. 1968;38:S61-7.

4. Nishimura RA, Otto CM, Bonow RO, Carabello BA, Erwin JP 3rd, Guyton RA, et al. American College of Cardiology;2014 AHA/ACC guideline for the management of patients with valvular heart disease: a report of the American College of Cardiology/American Heart Association Task Force on Practice Guidelines. J Thorac Cardiovasc Surg. 2014;21;64:1763.

5. Abelha FJ, Botelho M, Fernandes V, Barros H. Avaliação da qualidade de vida e mortalidade em pacientes com eventos cardíacos graves no pós-operatório. Rev Bras Anestesiol. 2010;60:268-84.

6. Christmann M, Costa C, Moussalle L. Avaliação da qualidade de vida de pacientes cardiopatas internados em um hospital público. Rev AMRIGS. 2011;55:239-43.

7. Dempster M, Donnelly $M$. Measuring the health related quality of life of people with ischaemic heart disease. Heart. 2000;83:641-4.

8. Gierlaszyńska K, Pudlo R, Jaworska I, Byrczek-Godulak K, Gąsior M. Tools for assessing quality of life in cardiology and cardiac surgery. tos de QVRS como rotina na prática clínica. Na realidade, estudos de medição da QVRS assumem cada vez mais importância em todas as áreas da medicina, uma vez que hoje em dia a mortalidade e morbilidade de qualquer tratamento não são suficientes para monitorizar os resultados. Os resultados a longo prazo, o impacto na QVRS, a taxa de recidivas e os efeitos secundários e sequelas das diversas terapêuticas assumem uma grande importância numa visão de valor criado pelos cuidados de saúde.

Em investigação futura, sugere-se a realização de estudos prospetivos multicêntricos e a longo prazo para identificação de variáveis preditoras dos resultados pós-operatórios da QVRS, que possam vir a possibilitar um melhor aconselhamento dos doentes.

\section{PROTEÇÃO DE PESSOAS E ANIMAIS}

Os autores declaram que os procedimentos seguidos estavam de acordo com os regulamentos estabelecidos pelos responsáveis da Comissão de Investigação Clínica e Ética e de acordo com a Declaração de Helsínquia da Associação Médica Mundial.

\section{CONFIDENCIALIDADE DOS DADOS}

Os autores declaram ter seguido os protocolos do seu centro de trabalho acerca da publicação de dados. Consentimento do doente obtido.

\section{CONFLITOS DE INTERESSE}

Os autores declaram não terem qualquer conflito de interesse relativamente ao presente artigo.

\section{FONTES DE FINANCIAMENTO}

Os autores declaram não ter recebido subsídios ou bolsas para a elaboração do artigo.

O Centro de Estudos e Investigação em Saúde da Universidade de Coimbra é financiado pela Fundação para a Ciência e a Tecnologia (UID/Multi/04066/2013).

9. Guyatt G. Measurement of health-related quality of life in heart failure. JACC Clin Electrophysiol. 1993;II:185A-91.

10. Gelsomino S, Lorusso R, Livi U, Masullo G, Lucà F, Maessen J, et al. Cost and cost-effectiveness of cardiac surgery in elderly patients $\mathrm{J}$ Thorac Cardiovasc Surg. 2011;142:1062-73.

11. Gjeilo KH, Klepstad P, Wahba A, Lydersen S, Stenseth R. Chronic pain after cardiac surgery: a prospective study. Acta Anaesthesiol Scand. 2010;54:70-8.

12. Gjeilo KH, Wahba A, Klepstad P, Lydersen S, Stenseth R. Survival and quality of life in an elderly cardiac surgery population: 5-year follow-up. Eur J Cardiothorac Surg. 2013;44:e182-8.

13. Goldsmith IR, Lip GY, Patel RL A prospective study of changes in patients' quality of life after aortic valve replacement. J Heart Valve Dis. 2001;10:346-53.

14. Shan L, Saxena A, McMahon R, Wilson A, Newcomb A. A systematic review on the quality of life benefits after aortic valve replacement in the elderly. J Thorac Cardiovasc Surg. 2013;145:1173-89.

15. Ferreira A, Nunes A, Rodrigues E, Camarneiro A. Qualidade de vida em doentes submetidos a cirurgia valvular cardíaca. Psicol Saúde Doenças. 2008;9:155-64.

16. Oliveira SM, Correia AS, Paiva M, Gonçalves A, Pereira M, Alves E, et al. Long-term survival, autonomy, and quality of life of elderly patients 
undergoing aortic valvereplacement. J Card Surg. 2012;27:20-3.

17. Ware J, Sherbourne C. The MOS 36-item Short-Form Health Survey (SF-36). Conceptual framework and item selection. Med Care. 1992;30:473-83.

18. Ware J, Snow K, Kosinski M, Gandek B. SF-36 Health survey manual and interpretation guide. Boston: The Health Institute; 1993.

19. Ferreira PL, Ferreira LN, Pereira LN. Medidas sumário física e mental de estado de saúde para a população portuguesa. Rev Port Saúde Pública. 2012;30:163-71.

20. Ware JE, Kosinski M, Keller SD. SF-36 physical and mental health summary scales: a user's manual. Boston: Health Institute; 1994.

21. Hawthorne G, Osborne R, Taylor A, Sansoni J. The SF36 Version 2: critical analyses of population weights, scoring algorithms and population norms. Qual Life Res. 2007;16:661-73.

22. Hopman WM, Towheed T, Anastassiades T, Tenenhouse A, Poliquin S, Berger $\mathrm{C}$, et al. Canadian Normative data for the SF-36 health survey. CMAJ. 2000;168:265-71.

23. Bartsch LJ, Butterworth P, Byles JE, Mitchell P, Shaw J, Anstey KJ. Examining the SF-36 in an older population: analysis of data and presentation of Australian adult reference scores from the Dynamic Analyses to Optimise Ageing (DYNOPTA) project. Qual Life Res. 2011;20:1227-36.

24. Juniper E, Norman G, Cox F, Roberts J. Comparison of the standard gamble, rating scale, AQLQ and SF-36 for measuring quality of life in asthma. Eur Respir J. 2001;18:38-44

25. Soto Torres M, Márquez Calderón $S$, Ramos Díaz I, Barba Chacón A, López Fernández F, Failde Martínez I. Health-related quality of life in coronary heart disease compared to norms in Spanish population. Qual Life Res. 2004;13:1401-07.

26. Ferreira PL. Criação da versão portuguesa do MOS SF-36. Parte I Adaptação cultural e linguística. Acta Med Port. 2000;13:55-66.

27. Ferreira PL. Criação da versão portuguesa do MOS SF-36. Parte II Testes de validação. Acta Med Port. 2000;13:119-27.

28. Ware J, Kosinski M, Dewey J. How to score Version Two of the SF-36 Health Survey. Rhode Island: QualityMetric, Incorporated; 2000.

29. Ferreira PL, Santana P. Percepção de estado de saúde e de qualidade de vida da população activa: contributo para a definição de normas portuguesas. Rev Port Saúde Pública. 2003;21:15-30.

30. Norman R, Church J, van den Berg J, Goodall S. Australian healthrelated quality of life population norms derived from the SF-6D. Aust N Z J Public Health. 2013;37:17-23.

31. Kosinki M, Kujawki S, Martin R, Wanke L, Buatti M, Ware J, et al. Healthrelated quality of life in early rheumatoid arthritis: pmpact of disease and treatment response. Am J Manag Care. 2002;8:231-40.
32. Oppe M, Charro F. Population norms and their uses. In: Szende A Williams A, editors. Measuring self-reported population health: an international perspective based on EQ-5D. Budapest: SpringMed Publishing; 2004.

33. Brazier J, Robert J, Deverill M. The estimation of a preference-based measure of health from the SF-36. J Health Econ. 2002;21:271-92.

34. Lam C, Brazier J, McGhee S. Valuation of the SF-6D Health States Is Feasible, Acceptable, Reliable, and Valid in a Chinese Population. Value Health. 2008;11:295-303.

35. Brazier J, Fukahara S, Roberts J, Kharroubi S, Yamamoto Y, Ikeda S, et al. Estimating a preference-based index from the Japanese SF-36. J Clin Epidemiol. 2009;62:1323-31.

36. Cruz L, Camey S, Hoffmann J, Rowen D, Brazier J, Fleck M, et al. Estimating the SF-6D value set for a population-based sample of Brazilians. Value Health. 2011;14:S108-14.

37. Abellán-Perpiñán JM, Sánchez Martínez FI, Martínez Pérez JE, Mendez I. Lowering the "floor" of the SF-6D scoring algorithm using a lottery equivalent method. Health Econ. 2012;21;1271-85.

38. Norman R, Viney R, Brazier J, Burgess L, Cronin P, King M, et al. Valuing SF-6D health states using a discrete choice experiment. Med Decis Making. 2014;34:773-86.

39. Ferreira PL, Ferreira LN. A medição de preferências em saúde na população portuguesa. Rev Port Saúde Pública. 2006;24:5-14.

40. Ferreira L, Ferreira P, Pereira L, Brazier J, Rowen D. A Portuguese value set for the SF-6D. Value Health. 2010;13:624-30.

41. van den Berg J. SF-6D population norms. Health Econ. 2012;21:150812.

42. Ferreira PL, Ferreira LN, Pereira LN. SF-6D portuguese population norms. Eur J Health Econ. 2015;16:235-41.

43. INE. Census 2011 Resultados definitivos - Portugal. Lisboa: INE; 2012.

44. Chocron S, Etievent JP, Viel JF, Dussaucy A, Clement F, Alwan K, et al. Prospective study of quality of life before and after open heart operations. Ann Thorac Surg. 1996;61:153-7.

45. Falcoz PE, Chocron S, Stoica L, Kaili D, Puyraveau M, Mercier M, et al. Open heart surgery: one-year self-assessment of quality of life and functional outcome. Ann Thorac Surg. 2003;76:1598-604.

46. Falcoz PE, Chocron S, Laluc F, Puyraveau M, Kaili D Mercier M, et al. Gender analysis after elective open heart surgery: a two-year comparative study of quality of life. Ann Thorac Surg. 2006;81:1637-43.

47. Gjeilo KH, Wahba A, Klepstad P, Lydersen S, Stenseth R. The role of sex in health quality of life after cardiac surgery: a prospective study. Eur J Cardiovascular Prev Rehabil. 2008;15:448-52.

48. Porter ME. What is value in health care? N Engl J Med. 2010;363:247781. 